\title{
Capacidade Funcional de Idosas de Instituição de Longa Permanência no Município de João Pessoa, Paraíba, Brasil: Estudo Piloto
}

\author{
Functional Capacity of Elderly from a Long-term Institution in the City \\ of João Pessoa, Paraíba, Brazil: A Pilot Study
}

\author{
ELIANE ARAÚJO DE OLIVEIRA ${ }^{1}$ \\ RONIELISON RIBEIRO DASILVA ${ }^{2}$ \\ ANTONIO GERALDO CIDRÃO DE CARVALHO ${ }^{3}$ \\ SANDRA MARIA CORDEIRO ROCHA DE CARVALHO ${ }^{4}$ \\ LAIRTON FABRÍCIO DE MENEZES MACIEL ${ }^{2}$ \\ ERIKA VALESKA DA COSTA ALVES ${ }^{2}$ \\ SANDRA EMÍLIA BENÍCIO BARROS 5 \\ MARIA DE FÁTIMAALCÂNTARA BARROS ${ }^{3}$
}

\section{RESUMO}

Objetivo: Analisar a funcionalidade e grau de independência/ dependência na realização das seis atividades de vida diária (vestir-se, banhar-se, alimentar-se, capacidade de transferência, continência e higiene pessoal) de idosas residentes numa Instituição de Longa Permanência. Material e Métodos: Estudo observacional, com medidas pré e pós. A amostra foi composta por 29 idosas $(80 \pm 10,4$ anos), das quais a maioria apresentou o tempo de institucionalização acima de 24 meses. Para coleta das variáveis utilizou-se o Índex de Independência nas Atividades de Vida Diária de Katz. Foi realizada a análise estatística descritiva e após o teste de Wilcoxon para analisar diferenças nas seis atividades básicas da vida diária, antes e depois de três meses $(p<0,05)$ e Correlação de Spearman para as variáveis idade e tempo de institucionalização. Resultados: Analisandose cada atividade básica da vida diária antes e depois de três meses, observou-se diminuição significativa da funcionalidade $(p<0,05)$ para todas as atividades básicas, exceto para vestir-se. Quando a amostra é categorizada como dependente ou independente, observou-se aumento significativo da dependência $(p<0,03)$. Conclusão: Houve um aumento na dependência das idosas e uma associação positiva entre as variáveis tempo de institucionalização e a idade.

\section{DESCRITORES}

Idoso. Instituição de Longa Permanência. Aptidão Física.

\begin{abstract}
Objective: To analyze the functionality and degree of independence / dependence in performing six activities of daily living (dressing, bathing, eating, ability to transfer, continence and personal hygiene) of elderly residents in a Long-term Institution. Material and Methods: This was an observational study with pre and post measures. The sample consisted of 29 elderly ( $80 \pm 10.4$ years), most of whom had the time of institutionalization over 24 months. For collection of variables, it was used the Index of Independence in Activities of Daily Living by Katz. Statistical descriptive analysis was employed, and the Wilcoxon test was used to analyze differences in the six basic activities of daily living before and after three months $(p<0.05)$; Spearman correlation was applied for the variables age and length of institutionalization. Results: After analyzing each basic activity of daily living before and after three months, it was observed a significant decrease in functionality $(p<0.05)$ for all basic activities, except dressing up. When the sample was categorized as dependent or independent, it was observed a significant increase in dependency $(p<0.03)$. Conclusion: There was an increase in elderly dependency and a positive association between the variables time of institutionalization and age.
\end{abstract}

\section{DESCRIPTORS}

Aged. Homes for the Aged. Physical Fitness.

1 Professora da Universidade Federal da Paraíba - Departamento de Fisioterapia/ Universidade Federal da Paraíba. Laboratório de Estudos do Envelhecimento Humano/ Núcleo de Pesquisa em Ciências do Movimento Humano/ do Centro de Ciências da Saúde/ Universidade Federal da Paraíba (CCS/UFPB), João Pessoa/PB, Brasil.

2 Fisioterapeuta, Graduado(a) pela Universidade Federal da Paraíba (UFPB), João Pessoa/PB, Brasil.

3 Professor(a) Ph.D. do Departamento de Fisioterapia da Universidade Federal da Paraíba e pesquisador do Laboratório de Fisioterapia em Saúde Coletiva (LabFISC) do Núcleo de Estudos e Pesquisas Epidemiológicas em Fisioterapia e Saúde (NEPEFIS) do Centro de Ciências da Saúde da Universidade Federal da Paraíba (CCS/UFPB), João Pessoa/PB, Brasil.

4 Professora Mestre em Educação do Departamento de Fisioterapia da Universidade Federal da Paraíba (UFPB), João Pessoa/PB, Brasil.

5 Professora Doutora em Ciências da Motricidade do Departamento de Fisioterapia da Universidade Federal da Paraíba (UFPB), João Pessoa/PB, Brasil. 
$\mathrm{O}$ envelhecimento pode ser entendido como um processo dinâmico e progressivo, no qual ocorrem modificações morfológicas, fisiológicas, bioquímicas e psicológicas, que determinam perda progressiva da capacidade de adaptação do indivíduo ao meio ambiente, ocasionando maior vulnerabilidade e maior incidência de processos patológicos (CARVALHO FILHO, 2007).

No Brasil tem se observado que a população com mais de 60 anos passou de 4,1\%, em 1940, para 8,6\% em 2000. Nas duas últimas décadas, a faixa etária que mais cresceu na população brasileira foi o "muito idoso", de 80 anos ou mais. Conforme o estudo feito em 2007 e 2008 pelo Instituto de Pesquisa Econômica Aplicada - IPEA (2008) existem 3.548 instituições de longa permanência (ILPI) no território brasileiro com 83.870 idosos, isso significa apenas $0,5 \%$ da população idosa. O idoso de uma ILPI costuma ter uma dependência física e mental mais elevada, quando bem de saúde, continua a morar com a família ou sozinho.

A independência dos idosos na execução das atividades de vida diária tem um impacto significativo na saúde e na qualidade de vida, sendo a perda da capacidade funcional o principal motivo para o ingresse numa ILPI (ARAÚJO et al., 2007). Segundo DIAS (2007), a diminuição da capacidade funcional é influenciada por fatores demográficos, socioeconômicos e culturais e pode estar relacionada com a presença de algumas enfermidades no processo de envelhecimento, causando dependência e perda de autonomia.

Dentre as alterações fisiológicas do envelhecimento observa-se a rigidez das cápsulas articulares e dos ligamentos, interferindo na amplitude articular acarretando movimentos mais lentos e imprecisos do idoso (BERLEZI et al., 2006). Também ocorre diminuição da massa muscular tornando o idoso mais frágil, gerando instabilidade e comprometimento da capacidade funcional, perda parcial ou total da independência (FITTS, 2003) e maior riscos de quedas com limitações de força muscular, equilíbrio, marcha e mobilidade (BONARDI, SOUZA, MORAES, 2007).

Por meio da avaliação da capacidade funcional, podem ser definidas estratégias de promoção de saúde dos idosos, visando retardar ou prevenir as incapacidades. A avaliação funcional pode ser definida como uma tentativa sistematizada de medir, de forma objetiva, os níveis em que uma pessoa é capaz de desempenhar determinadas atividades ou funções em diferentes áreas, utilizando-se de habilidades diversas para o desempenho das tarefas da vida cotidiana (SCHNEIDER, MARCOLIN, DALACORTE, 2008).

Muitos são os instrumentos utilizados para avaliação funcional em gerontologia, todavia o Índex de Dependência nas Atividades de Vida Diária desenvolvido por KATZ (1963) é o mais utilizado nos estudos gerontológicos nacionais e internacionais devido à sua praticidade de aplicação, especificidade e confiabilidade na mensuração da realização de seis atividades de vida diária.

Como a incapacidade funcional afeta cerca de um quarto dos idosos, sendo em maior proporção as mulheres, identificar e reabilitar os idosos são fatores de extrema importância para mantê-los mais saudáveis e independentes dentro das possibilidades terapêuticas (PARAHIBA, SIMÕES, 2006; CARVALHO, PEIXOTO, CAPELLA, 2007). Segundo CALDAS (2003), a dependência não é um estado permanente, mas um processo dinâmico, cuja evolução pode ser modificada, prevenida e/ou reduzida, denotando-se a necessidade de atenção no que diz respeito ao grau de dependência para as atividades de vida diária.

Considerando que o processo de envelhecimento aliado à institucionalização influencia na limitação da capacidade funcional, o objetivo deste estudo foi analisar a funcionalidade e o grau de independência/dependência na realização de seis atividades de vida diária num grupo de idosas residentes numa Instituição de Longa Permanência, localizada na cidade de João Pessoa/PB, tendo como instrumento de avaliação funcional o Índex de Independência nas Atividades de Vida Diária de Katz.

\section{MATERIAL E MÉTODOS}

Trata-se de um estudo piloto, de caráter descritivo, observacional e de seguimento, por conveniência, com uma medida no início do estudo e uma medida após três meses. A população do estudo foi composta por 32 idosas institucionalizadas, numa Instituição de Longa Permanência, localizada na cidade de João Pessoa, Paraíba, Brasil. Observou-se que nos prontuários analisados não havia relato de algumas variáveis importantes que poderiam ser acrescentadas na pesquisa, tais como: diagnóstico clínico, profissão e motivo da institucionalização.

Como critério de inclusão no estudo, as idosas deveriam residir na instituição de longa permanência e deveriam ter idade igual ou superior a 60 anos. O critério de exclusão foi o óbito de idosas antes da finalização da pesquisa. Neste sentido, os dados de três não foram aproveitados, dois por motivo de óbito e um por apresentar idade abaixo de 60 anos sendo considerado, portanto, para a amostra do estudo $n=29$ sujeitos com idade média de ( $80 \pm 10,4$ anos).

O trabalho foi aprovado pelo Comitê de Ética em 
Pesquisa do Hospital Universitário Lauro Wanderley de acordo com a Resolução 196/96 do Conselho Nacional de Saúde (Protocolo CEP/HULW nº 594/10). Todos os participantes do estudo assinaram o Termo de Consentimento Livre e Esclarecido.

Os dados foram coletados no período de novembro de 2010 a março de 2011, por meio de consulta aos prontuários, além de entrevistas com os cuidadores das idosas, utilizando-se os seguintes instrumentos: 1) Prontuário das residentes, observando-se as seguintes variáveis: idade, cor, nível de escolaridade e tempo de permanência na instituição até a data da coleta de dados; 2) Índice de Katz, aplicado por meio das observações dos pesquisadores e complementadas com informações dos cuidadores das idosas. Os referidos dados foram colhidos no início do estudo (medida antes) e uma medida após três meses (medida pós), sem nenhuma intervenção fisioterapêutica, sendo abordado o nível de funcionalidade das idosas no que diz respeito às seis atividades básicas da vida diária como: tomar banho; vestir-se; alimentar-se; realizar higiene pessoal; transferências e continência. Para classificar a funcionalidade mediante a realização de tais atividades, categorizou-se como independente os sujeitos capazes de realizar as seis atividades e como dependente os sujeitos incapazes de realizar uma ou mais atividades básicas da vida diária, necessitando de algum tipo de ajuda.

Realizou-se a estatística descritiva, como frequência absoluta e relativa por meio do cálculo de medidas de tendência central (média aritmética) e de dispersão (desvio padrão) para as variáveis contínuas e de frequência para as variáveis categóricas. Para o teste de hipótese, foi pré-fixado o nível de significância de $5 \%$. Foi realizado o teste de normalidade Shapiro Wilk, seguindo com o teste estatístico de Wilcoxon (teste pareado) para analisar as diferenças nas seis atividades básicas da vida diária, antes e depois de três meses, sem intervenção fisioterapêutica. Foi realizada ainda, a correlação Rho de Spearman, para estudos não paramétricos, com as variáveis idade e tempo de institucionalização. Os dados foram organizados e analisados utilizando-se o Programa SPSS (Statistical Package for Social Sciences), versão 19.0 para Windows.

\section{RESULTADOS}

A maioria dos sujeitos deste estudo apresentou: idade acima de 80 anos $(80,45 \pm 10,4)$, sendo a mínima de 62 anos e a máxima 105 anos; são analfabetas $(72,4 \%)$; cor branca $(51,7 \%)$ e residem na instituição há mais de 2 anos; institucionalizadas por diversos motivos como: não terem familiares com quem morar; apesar de terem os familiares, os mesmos não podem cuidar e terem familiares que não querem cuidar.

Analisando o tempo de permanência na referida Instituição, observou-se uma grande variação no tempo de institucionalização, mínimo de 01 mês e máximo de 10 anos. Verificou-se também que quanto maior a faixa etária, maior o tempo de institucionalização, cuja correlação foi bastante significativa (Tabela 1).

Analisando-se separadamente cada atividade básica da vida diária antes e depois de três meses por meio do Índice de Katz, observou-se uma diminuição da funcionalidade bastante significativa $(\mathrm{p}<0,05)$ para todas as atividades básicas, exceto para vestir-se, conforme Tabela 2.

Tabela 1. Correlação entre a faixa etária das idosas e o tempo de institucionalizaç̃o em intervalos de 6 meses

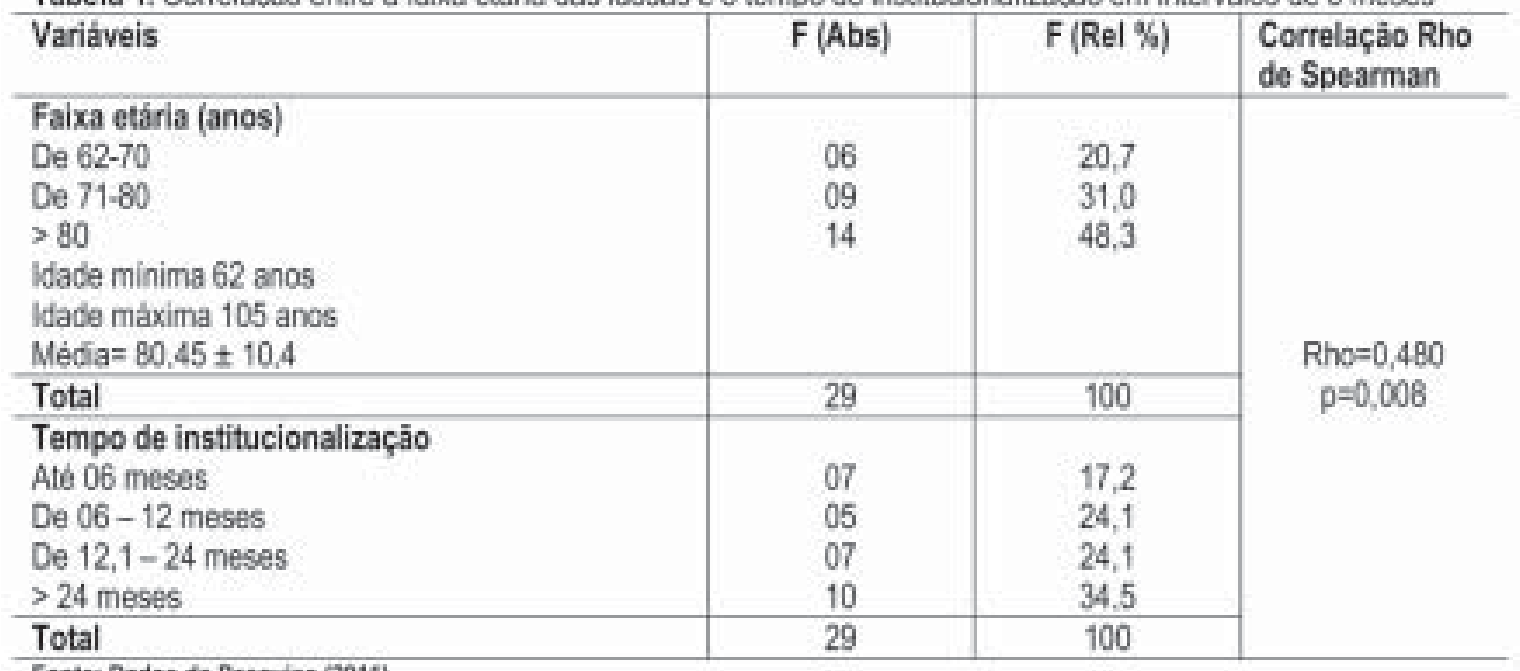

Fonte: Dados da Pescuisa (2011). 
Tabela 2. Descrição da dependência e independència nas atividades básicas da vida diária antes e depois de 03 meses

\begin{tabular}{|c|c|c|c|c|c|c|}
\hline \multirow{3}{*}{ Variáveis } & \multicolumn{2}{|c|}{ ANTES } & \multicolumn{2}{|c|}{ DEPOIS } & \multirow{2}{*}{\multicolumn{2}{|c|}{$\begin{array}{l}\text { Teste de Wilcoxon } \\
\text { (Antes e Depois) }\end{array}$}} \\
\hline & \multirow{2}{*}{$\begin{array}{l}\text { Não } \\
\text { Realiza } \\
f(\%)\end{array}$} & \multirow{2}{*}{$\begin{array}{l}\text { Realiza } \\
f(\%)\end{array}$} & \multirow{2}{*}{$\begin{array}{l}\text { Realiza } \\
f(\%)\end{array}$} & \multirow{2}{*}{$\begin{array}{l}\text { Näo } \\
\text { Realiza } \\
\text { f }(\%)\end{array}$} & & \\
\hline & & & & & $\mathbf{Z}$ & $\mathbf{P}$ \\
\hline Banho & $15(51,7)$ & $14(48,3)$ & $6(20.7)$ & $23(79,3)$ & $-2,236$ & 0.025 \\
\hline Vestir-se & $15(51,7)$ & $14(48.3)$ & $8(27,6)$ & $21(72.4)$ & $-1,732$ & 0.083 \\
\hline Hiqiene Pessoal & $15(51,7)$ & $14(48.3)$ & $5(17.2)$ & $24(82,8)$ & $-2,449$ & 0.014 \\
\hline Transferências & $14(48.3)$ & $15(51,7)$ & $5(17,2)$ & $24(82,8)$ & $-3,606$ & 0.001 \\
\hline Continència & $14(48,3)$ & $15(51,7)$ & $9(31.0)$ & $20(69.0)$ & $-2,714$ & 0.007 \\
\hline Alimentacto & $12(41.4)$ & $17(58.6)$ & $6(20.7)$ & $23(79.3)$ & -3.500 & 0.001 \\
\hline
\end{tabular}

Fonte: Dados da Pesquisa [2011).

Ao categorizar a amostra como dependente ou independente, antes e depois de três meses sem intervenção fisioterapêutica observou-se um aumento da dependência bastante significativo $(Z=-2,236$; $\mathrm{p}=0,025)$.

\section{DISCUSSÃO}

A capacidade funcional é definida como a habilidade física e mental para manter uma vida independente e autônoma com a realização de uma tarefa ou ação pelo indivíduo (AVILA-FUNES, GRAYDONALD, PAYETTE, 2006). Segundo ROSA et al. (2003), é denominada "incapacidade funcional" a dificuldade ou impossibilidade no desempenho de determinadas atividades ou tarefas básicas da vida cotidiana.

No estudo de MARCHON, CORDEIRO, NAKANO (2010) ao analisarem a eficácia da Short Physical Performance Balance, uma bateria de testes para detectar alterações na capacidade funcional do idoso institucionalizado, observaram que muitos idosos se consideravam ativos e satisfeitos com o lazer, mas ao analisarem a capacidade funcional no decorrer do tempo foi verificada perda funcional. Em estudo realizado por CARVALHO, PEIXOTO e CAPELLA (2007) utilizandose o Índice de Independência de Katz, observou-se que $50 \%$ dos idosos apresentaram dependência importante. Resultados semelhantes foram observados no presente estudo, no qual as idosas investigadas apresentaram após três meses, um aumento no nível de dependência para todas as atividades, bastante significativo.
Em estudo realizado por ARAÚJO e CEOLIM (2007) foi avaliado o grau de independência de 187 idosos residentes em três Instituições de Longa Permanência na Cidade de Taubaté-SP, através do Índice de Independência de Katz. Neste estudo observou-se que 70 idosos foram considerados independentes, porém durante a reavaliação 5 meses depois, 13 idosos anteriormente classificados em grau A de independência, apresentavam-se em estado de dependência funcional e cognitiva ou haviam falecido.

Com relação ao tempo de institucionalização do presente estudo, percebe-se que com o passar dos meses/anos a capacidade funcional fica prejudicada, corroborando com o estudo de PAVARINI (1996) onde afirma que nas instituições asilares de longa permanência, a dependência física é muitas vezes estimulada, pois os próprios funcionários preferem ajudar os idosos nas suas atividades, quando esses já apresentam inabilidade para executar tarefas simples, embora não sejam incapazes para fazê-las. A institucionalização representa um fator de risco, os idosos institucionalizados na maioria são fragilizados e apresentam morbidades físicas ou mentais. Pelo seu isolamento social, inatividade física e processos psicológicos subentende-se que quanto maior o tempo de institucionalização, maior a debilidade do idoso (SANTOS, ANDRADE, 2005).

MAZUIM (2005), em estudo com idosos lúcidos constatou que, antes de serem institucionalizados, tanto os homens quanto as mulheres realizavam atividades rotineiras, o que favorecia a manutenção da autonomia e independência; após a institucionalização, em virtude da escassa atividade sugerida, muitos perdiam a capacidade funcional. 
AIRES, PAZ, PEROSA (2006) ao avaliarem as atividades de vida diária por meio do Índice de Katz em dez idosos institucionalizados, constataram que as Atividades de Instrumentais de Vida Diária (AIVDs) são as primeiras a serem excluídas do cotidiano do idoso institucionalizado e, dessa forma, a incapacidade funcional para as AIVDs compromete a saúde mental do idoso e, conseqUentemente, a socialização.

\section{CONCLUSÃO}

Este estudo demonstrou que a dependência de idosas está diretamente ligada ao tempo de institucionalização e à idade. Com relação à capacidade funcional, observou-se uma menor dependência na alimentação, enquanto que as atividades de banhar-se, vestir-se, e higiene pessoal são as que apresentaram uma maior frequência de dependência de auxilio. Analisando-se as atividades básicas da vida diária antes e depois de três meses, observou-se uma diminuição da funcionalidade bastante significativa para todas as atividades básicas, exceto para a atividade de "vestirse".

\section{REFERÊNCIAS}

1. AIRES M, PAZ AD, PEROSACT. O grau de dependência e características de pessoas idosas institucionalizadas. Rev Bras Ciênc Envelhec Hum. 2006; 3(2), 79-91.

2. ARAÚJO F, RIBEIRO JLP, OLIVEIRA A, PINTO C. Validação da escala de Lawton e Brody numa amostra de idosos não institucionalizados. Rev Port Saúde Pública. 2007; 25(2):59-66.

3. ARAÚJO MOPH, CEOLIM MF. Avaliação do grau de independência de idosos residentes de longa permanência. Rev ESC Enferm USP. 2007; 41(3):378385.

4. AVILA-FUNES JA, GRAY-DONALD K, PAYETTE H. Medicíon de las capacidades físicas de adultos mayores de Québec: un análisis secundario del estudio NuAge. Salud Publica Mex. 2006; 48(6):446-454.

5. BERLEZI EM, ROSA PV, SOUZAACA, SCHNEIDER RH. Comparação antropométrica e do nível de aptidão física de mulheres acima de 60 anos praticantes de atividade física regular e não praticantes. Rev Bras Geriatr Gerontol. 2006; 9(3):1-13.

6. BONARDI G, SOUZA VBA, MORAES JFD. Incapacidade funcional e idosos: Um desafio para os profissionais da saúde. Rev. Sci Med. 2007; 17(3):138-144.

7. CALDAS CP. Envelhecimento com dependência: responsabilidades e demandas da família. Cad Saúde Pública. 2003; 19(3):773-781.

8. CARVALHO GA, PEIXOTO NM, CAPELLA PD. Análise comparativa da avaliação funcional do paciente geriátrico institucionalizado por meio dos protocolos de Katz e Tinetti. Lecturas Educación Física y Deportes. 2007; 114:1-5.

9. CARVALHO FILHO ET. Fisiologia do envelhecimento. In: PAPALÉO NETO, M. Tratado de Gerontologia. 2aed. São Paulo: Editora Athneu, 2007. cap.8, p.105-120.
10. DIAS AM. O processo de envelhecimento humano e a saúde do idoso nas práticas curriculares do curso de fisioterapia da UNIVALI, campus Itajaí: um estudo de caso, [Dissertação de Mestrado]. Vale do Itajaí: Universidade do Vale do Itajaí; 2007. 189p.

11. FITTS R. Effects of regular exercise training on skeletal muscle contractile function. Am J Phys Med Rehab. 2003; 82(4): 320-331.

12. INSTITUTO DE PESQUISAECONÔMICAAPLICADA- IPEAPensando no futuro dos idosos/2008. Disponível em: <www.ipea.gov.br> Acesso em: 14 jun. 2012.

13. KATZ S, FORD AB, MOSKOWITZ RW, JACKSON BA, JAFFE MW. Studies of illness in the aged. The index of ADL: a standardized measure of biological and psychosocial function. JAMA. 1963; 185(12):914-919.

14. MARCHON RM, CORDEIRO RC, NAKANO NM. Capacidade Funcional: estudo prospectivo em idosos residentes em uma instituição de longa permanência. Rev Bras Geriatr Gerontol. 2010; 13(2):203-214.

15. MAZUIM CR. Idoso institucionalizado: suporte, abrigo ou segregação? $1^{\text {a }}$ ed. Canoas: Editora Ulbra, 2005, 34p.

16. PARAHIBA MI, SIMÕES CCS. A prevalência de incapacidade funcional em idosos no Brasil. Ciênc Saúde Coletiva. 2006; 11(4): 967-974.

17. PAVARINI SCI. Dependência comportamental na velhice: uma análise do cuidado prestado ao idoso institucionalizado, [Dissertação de Mestrado]. Campinas: Universidade Estadual de Campinas; 1996. 250p.

18. ROSA TEC, BENÍCIO MHD, LATORRE MRDO, RAMOS LR. Fatores determinantes da capacidade funcional entre idosos. Rev Saúde Pública. 2003; 37(1): 40-48. 
19. SANTOS MLC, ANDRADE MC. Incidência de quedas relacionada aos fatores de riscos em idosos institucionalizados. Rev Baiana Saúde Pública. 2005; 29(1):57-58. Disponível em: <http: www.scielo.br>. Acesso em: 10 ago 2006.

20. SCHNEIDER RH, MARCOLIN D, DALACORTE RR. Avaliação funcional de idosos. Sci Med. 2008; 18(1): 4-9.
Correspondência

Eliane Araújo de Oliveira

Laboratório de Estudos do Envelhecimento Humano/

Centro de Ciências da Saúde/ UFPB/ Campus I

João Pessoa - Paraíba - Brasil

58.000 .000

E-mail:elianeao@uol.com.br 